\title{
Scaleheights of 486 southern spiral galaxies and some statistical correlation $^{\star}$
}

\author{
J. Ma, Q.-H. Peng, R. Chen, Z.-H. Ji, and C.-P. Tu \\ Department of Astronomy, Nanjing University, Nanjing, 210093, China \\ Received January 2; accepted April 15, 1997
}

\begin{abstract}
Based on Peng's method (1988), we obtain scaleheights of 486 southern spiral galaxies, the images of which are taken from the Digitized Sky Survey ${ }^{1}$ at Xinglong Station of Beijing Astronomical Observatory. The fitted spiral arms of 70 galaxies are compared with their images to get their optimum inclinations. The scaleheights of other 416 ones are listed in Table A1 in Appendix. After compiling and analyzing the data, we find some statistical correlations. The most interesting results are that a flatter galaxy is bluer and looks brighter, and galaxies become flatter along the Hubble sequence Sab Scd.
\end{abstract}

Key words: galaxies: fundamental parameters galaxies: spiral - galaxies: structure

\section{Introduction}

The scaleheight of a galactic disk is certainly a very important attribute of a spiral galaxy. There are two wellknown approaches to estimate the parameter. The one suggested by van der Kruit \& Searle $(1981,1982)$ is for edge-on spirals. The other one is for non-edge-on spiral galaxies, which are the majority of spirals, proposed by Peng (1988). The latter is useful and rather simple as long as spiral arms are distinguishable on their images. As a test for his method, Peng measured the

Send offprint requests to: J. Ma, e-mail: qhpeng@nju.edu.cn

* Table A1 is available in electronic from only, via anonymous ftp 130.79.128.5 or http://cdsweb.u-strasbg.fr/Abstract.html

1 Based on photographic data of the National Geographic Society - Palomar Observatory Sky Survey (NGS-POSS) obtained using the Oschin Telescope Palomar Mountain. The NGS-POSS was funded by a grant from the National Geographic Society to the California Institute of Technology. The plates were processed into the present compressed digital form with their permission. The Digitized Sky Survey was produced at the Space Telescope Science Institute under US Government grant NAG W-2166. scaleheights of four spiral galaxies (NGC 628 (M 74), NGC 5236 (M 83), NGC 5194 (M 51), and UGC 2885).

The main points of Peng's method (1988) in present paper are:

1. Changing the grey-scale of the image of a galaxy, finding the position of the innermost point of the spiral arm and measuring its coordinates $\left(\rho_{0}, \theta_{0}\right)$ from the galactic center,

2. Assuming different inclinations $(\gamma$, i.e. the angle between the galactic plane and the tangent plane) near the value of $\arccos \left(d_{25} / D_{25}\right)$ and fitting the spiral arm starting from that innermost point with a logarithmic spiral curve to get its wounding parameter $(\Lambda)$,

3. Determining the optimum inclination and the corresponding wounding parameter by comparing the fitted spiral arm with the image. Figure 11 gives an example how to use Peng's method, we fit NGC 1096 with the optimum inclination $\left(\gamma=17.3^{\circ}\right)$ and wounding parameters ( $\Lambda_{1}=10.24$ and $\Lambda_{2}=9.82$, corresponding to two arms),

4. The scaleheight of the galactic disk, $h$ in arc-minute, may be calculated by

$$
\begin{aligned}
& h=\frac{2 r_{0}}{\sqrt{m^{2}+\Lambda^{2}}}, \\
& r_{0}=\frac{\rho_{0} \sqrt{\sin ^{2} \theta_{0}+\cos ^{2} \theta_{0} \cos ^{2} \gamma}}{\cos \gamma},
\end{aligned}
$$

where $m$ is the number of the arms.

5. The scaleheight of the galactic disk, $H$ in $\mathrm{kpc}$, is

$H=h d$,

here $d$ is the distance of the galaxy from the Galactic Center,

$d=\frac{v_{\mathrm{GSR}}}{H_{0}}$,

where $H_{0}$ is the Hubble constant taken as $75 \mathrm{~km} / \mathrm{s} / \mathrm{Mpc}$, $v_{\mathrm{GSR}}$, taken from the Third Catalog of Bright Galaxies by de Vaucouleurs et al. (1991, RC3), the weighted mean 
radial velocity of the radio and optical redshifts of the galaxy corrected to the Galactic Center.

This paper presents our estimation of scaleheights for 486 southern spiral galaxies selected from more than 1500 ones whose images are taken from the Digitized Sky Survey. All these galaxies are the grand design spiral ones with Arm Classification $\geq 5$ (Elmegreen \& Elmegreen 1987). Since there are usually two arms in a galaxy, the arm with the minimum $\rho_{0}$ is picked up for measuring the scaleheight.

Our data reduction and analyses were done on the Sun Workstation installed with IRAF software.

\section{Samples and measurement}

Our statistical sample contains 70 galaxies, selected from more than 500 southern spirals with $(B-V)_{T}^{0}$ (i.e. the total color indexes corrected for differential galactic and internal extinction (to "face-on") and redshift between $B$ and $V$ bands) in RC3 according to one single criterion: they have distinguishable spiral arms. The mean numerical Hubble stage indexes $(T)$ of these galaixes are from 2 to 6 , and $\log \left(D_{25} / d_{25}\right)$ less than 0.76. $D_{25}$ and $d_{25}$, taken from RC3, are the apparent major and minor isophotal diameters measured at or reduced to the surface brightness level $\mu_{B}=25.0 B-\mathrm{m} / \mathrm{ss}$. It is well known that $d_{25} / D_{25}$ is usually the approximate value of $\cos \gamma$. Following Peng's method, we have adjusted the value of $\gamma$ around $\arccos \left(d_{25} / D_{25}\right)$ in order to do the fitting well, meaning that the inclination of a galaxy may be derived by well-fitting the spiral arms with a logarithmic spiral curve on its image. The resulting inclinations for these galaxies are listed in Table 1. The errors of our estimation mainly come from: a) the position of starting point for an arm; b) the inclination of a galaxy; c) the position of the galactic center. The errors from the position of the starting point and of the center, however, can be decreased if the grey-scale of an image is modified properly by using IRAF to obtain the fine structure of a galaxy as deeply as we could have. The error estimations are derived from the formulae given by Peng (1988).

\section{Table of scaleheights of galaxies}

The scaleheights $(H)$ of 70 southern spiral galaxies and their relative errors are listed in Table 1. At the same time, some parameters of these galaxies are also included, where $m$ is the number of the arms in a galaxy, $T$ the mean numerical Hubble stage index of a galaxy, $\gamma$ the inclination of a galaxy, $\Lambda$ the wounding parameter of a spiral arm, $\mu$ $(=\arctan (m / \Lambda))$ the pitch angle of a spiral arm, $h$ the apparent scaleheight of a galaxy, $d$ the distance of a galaxy from the Galactic Center, $H / D_{0}$ the flatness of a galaxy ( $D_{0}$ is the isophotal major diameter corrected to the "faceon" $\left(\gamma=0^{\circ}\right)$, and for Galactic extintion to $A_{\mathrm{g}}=0$, but not for redshift).

\section{Statistical properties of flatness of spiral galaxies}

1. Dependence on color.

Figures 1 and 3 present the correlation between flatness of spiral galaxy $\left(H / D_{0}\right)$ and $(B-V)_{T}^{0}$, Figs. 2 and 4 show the correlation between the scaleheight of spiral $(H)$ and $(B-V)_{T}^{0}$. Results obtained by van der Kruit and Searle's method are also plotted in Figs. 3 and 4 for comparison. It is obvious that the results obtained by different methods are consistent.

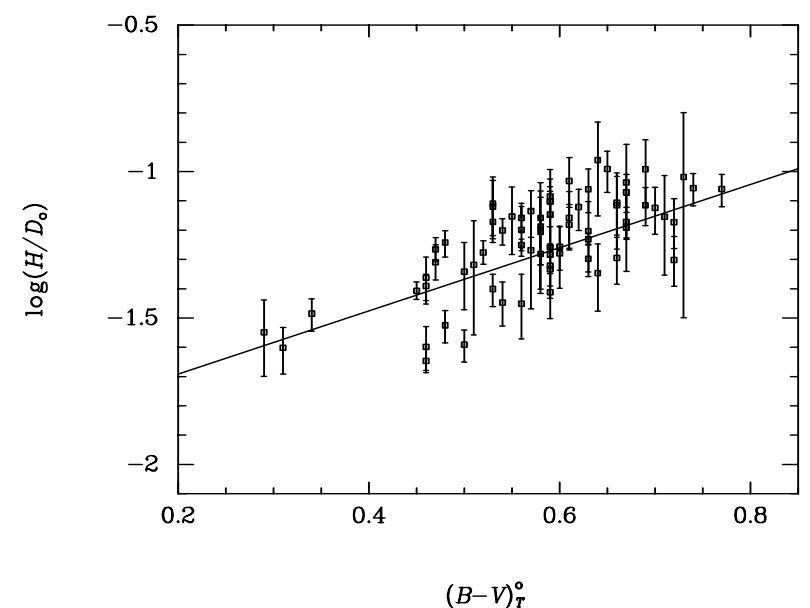

Fig. 1. Flatness of spiral galaxy plotted versus the corrected $B-V$ color

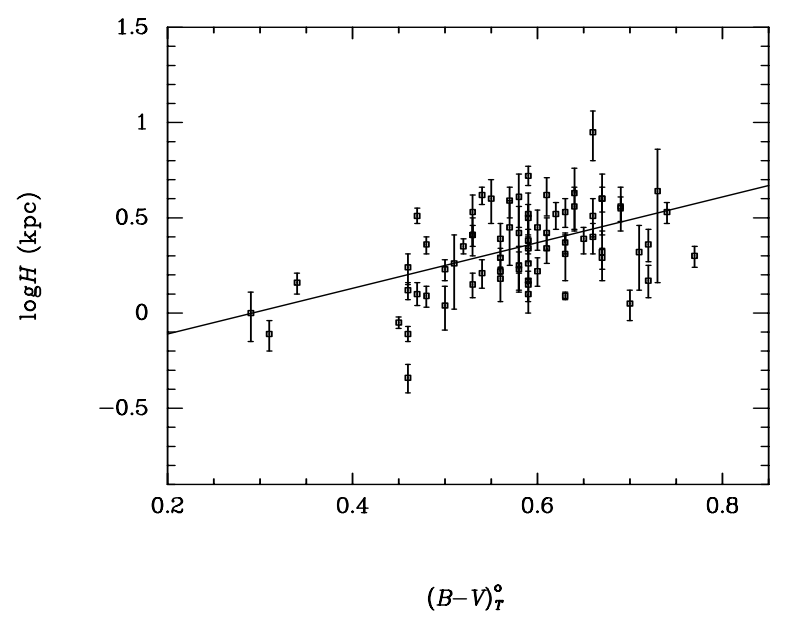

Fig. 2. Scaleheight of spiral galaxy plotted versus the corrected $B-V$ color

The equations of the regression are

$\log \left(H / D_{0}\right)=a(B-V)_{T}^{0}+b$

$\log H=a^{\prime}(B-V)_{T}^{0}+b^{\prime}$. 
Table 1. Scaleheights of 70 southern spiral galaxies

\begin{tabular}{|c|c|c|c|c|c|c|c|c|c|c|}
\hline $\mathrm{PGC}$ & Names & $m$ & $T$ & $\begin{array}{c}\gamma \\
\left(^{\circ}\right) \\
\end{array}$ & $\Lambda \pm \mathrm{d} \Lambda / \Lambda$ & $\begin{array}{c}\mu \\
\left(^{\circ}\right)\end{array}$ & $\begin{array}{c}h \pm \mathrm{d} h / h \\
\left({ }^{\prime}\right)\end{array}$ & $\begin{array}{c}d \\
(\mathrm{Mpc}) \\
\end{array}$ & $\begin{array}{c}H \pm \mathrm{d} H / H \\
(\mathrm{kpc})\end{array}$ & $H / D_{0}$ \\
\hline $\begin{array}{ll}\text { PGC } 382 \\
\end{array}$ & & 2 & 5.0 & 54.9 & $5.47 \pm 38.8 \%$ & 20.1 & $0.088 \pm 26.1 \%$ & 137.55 & $3.60 \pm 26.1 \%$ & 0.045 \\
\hline PGC 2052 & NGC 150 & 2 & 3.0 & 66.4 & $17.87 \pm 27.2 \%$ & 6.4 & $0.177 \pm 26.6 \%$ & 21.08 & $1.08 \pm 26.6 \%$ & 0.045 \\
\hline PGC 2437 & NGC 210 & 2 & 3.0 & 48.6 & $5.71 \pm 27.2 \%$ & 19.3 & $0.314 \pm 27.1 \%$ & 22.37 & $2.04 \pm 27.1 \%$ & 0.063 \\
\hline PGC 2595 & NGC 238 & 2 & 3.1 & 35.6 & $13.81 \pm 10.7 \%$ & 8.2 & $0.126 \pm 10.2 \%$ & 113.91 & $4.17 \pm 10.2 \%$ & 0.063 \\
\hline PGC 3089 & NGC 289 & 2 & 4.0 & 50.9 & $7.56 \pm 30.2 \%$ & 14.8 & $0.338 \pm 29.8 \%$ & 21.48 & $2.11 \pm 29.8 \%$ & 0.064 \\
\hline PGC 3190 & & 4 & 4.2 & 51.9 & $13.43 \pm 25.1 \%$ & 16.6 & $0.069 \pm 24.4 \%$ & 139.99 & $2.81 \pm 24.4 \%$ & 0.053 \\
\hline PGC 5269 & NGC 539 & 2 & 5.0 & 44.7 & $9.46 \pm 31.9 \%$ & 11.9 & $0.110 \pm 30.7 \%$ & 128.80 & $4.12 \pm 30.7 \%$ & 0.069 \\
\hline PGC 7379 & NGC 782 & 2 & 3.0 & 29.5 & $9.32 \pm 19.3 \%$ & 12.1 & $0.172 \pm 18.6 \%$ & 78.33 & $3.92 \pm 18.6 \%$ & 0.073 \\
\hline PGC 8012 & & 2 & 2.6 & 56.5 & $6.54 \pm 26.3 \%$ & 17.0 & $0.161 \pm 26.1 \%$ & 77.45 & $3.63 \pm 26.1 \%$ & 0.102 \\
\hline PGC 8451 & NGC 858 & 2 & 5.0 & 29.4 & $12.91 \pm 9.5 \%$ & 8.8 & $0.068 \pm 8.9 \%$ & 164.23 & $3.25 \pm 8.9 \%$ & 0.054 \\
\hline PGC 9420 & NGC 947 & 2 & 5.2 & 58.3 & $16.00 \pm 20.2 \%$ & 7.1 & $0.091 \pm 17.8 \%$ & 66.31 & $1.75 \pm 17.8 \%$ & 0.043 \\
\hline PGC 9426 & NGC 945 & 2 & 4.5 & 33.7 & $9.56 \pm 24.9 \%$ & 11.8 & $0.188 \pm 22.9 \%$ & 59.60 & $3.26 \pm 22.9 \%$ & 0.078 \\
\hline PGC 9747 & NGC 986 & 2 & 2.0 & 44.7 & $11.96 \pm 14.6 \%$ & 9.5 & $0.262 \pm 12.7 \%$ & 25.43 & $1.94 \pm 12.7 \%$ & 0.067 \\
\hline PGC 9973 & & 2 & 3.3 & 59.1 & $10.77 \pm 21.7 \%$ & 10.5 & $.094 \pm 17.9 \%$ & 115.59 & $3.16 \pm 17.9 \%$ & 0.055 \\
\hline PGC 10336 & NGC 1096 & 2 & 4.2 & 17.3 & $10.78 \pm 17.7 \%$ & 11.5 & $0.097 \pm 17.9 \%$ & 87.16 & $2.50 \pm 17.9 \%$ & 0.050 \\
\hline PGC 10870 & & 2 & 3.8 & 37.4 & $6.84 \pm 15.5 \%$ & 16.3 & $0.120 \pm 14.8 \%$ & 115.21 & $4.02 \pm 14.8 \%$ & 0.085 \\
\hline PGC 11691 & A0305-31 & 2 & 5.9 & 53.9 & $18.15 \pm 17.2 \%$ & 6.3 & $0.088 \pm 16.2 \%$ & 63.35 & $1.62 \pm 16.2 \%$ & 0.036 \\
\hline PGC 12412 & NGC & 2 & 4.0 & 48.6 & $10.69 \pm 19.2 \%$ & 10.6 & $0.245 \pm 18.3 \%$ & 19.95 & $1.42 \pm 18.3 \%$ & 0.039 \\
\hline PGC 13179 & NGC & 2 & 3.0 & 64.7 & $16.2 \%$ & 14.4 & $0.886 \pm 11.2 \%$ & 20.55 & $5.29 \pm 11.2 \%$ & 0.079 \\
\hline PGC 13586 & NGC 1433 & 2 & 2.0 & 56.4 & $4.80 \pm 14.7 \%$ & 22.6 & $0.562 \pm 13.5 \%$ & 12.27 & $2.00 \pm 13.5 \%$ & 0.087 \\
\hline PGC 14704 & IC 2050 & 2 & 3.0 & 37.4 & $15.26 \pm 14.9 \%$ & 7.5 & $0.051 \pm 14.6 \%$ & 162.65 & $2.41 \pm 14.6 \%$ & 0.048 \\
\hline PGC 14897 & NGC 1566 & 2 & 4.0 & 37.4 & $8.30 \pm 31.8 \%$ & 13.6 & $0.295 \pm 24.6 \%$ & 17.60 & $1.51 \pm 24.6 \%$ & 0.035 \\
\hline PGC 15850 & NGC 1640 & 2 & 3.0 & 39.1 & $8.13 \pm 18.9 \%$ & 13.8 & $0.198 \pm 18.5 \%$ & 19.71 & $1.13 \pm 18.5 \%$ & 0.075 \\
\hline PGC 15941 & NGC 1672 & 2 & 3.0 & 38.7 & & 12.8 & $70 \pm 4.7 \%$ & 15.40 & $6 \pm 4.7 \%$ & 0.056 \\
\hline PGC 16136 & & 2 & 4.5 & 43.1 & $\pm 29.4 \%$ & 12.1 & $0.095 \pm 37.3 \%$ & 76.21 & $2.11 \pm 37.3 \%$ & 0.070 \\
\hline PGC 16751 & A0504-17 & 2 & 5.0 & 52.9 & $10.56 \pm 24.4 \%$ & 10.7 & $0.108 \pm 19.9 \%$ & 58.41 & $1.83 \pm 19.9 \%$ & 0.071 \\
\hline PGC 17436 & NGC 1964 & 2 & 3.0 & 74.7 & $10.05 \pm 21.9 \%$ & 11.3 & $0.378 \pm 17.6 \%$ & 20.09 & $2.21 \pm 17.6 \%$ & 0.066 \\
\hline PGC 18092 & IC 2160 & 2 & 4.7 & 66.5 & $\pm 13.4 \%$ & 9.9 & $0.131 \pm 10.8 \%$ & 60.49 & $2.30 \pm 10.8 \%$ & 0.057 \\
\hline PGC 19413 & & 2 & 3.0 & 70.7 & $=29.3 \%$ & 6.9 & 0.1 & 31.79 & $1.77 \pm 25$ & 0.065 \\
\hline PGC 19498 & & 4 & 5.1 & 60.0 & $\pm 18.5 \%$ & 10.7 & $0.060 \pm 13.4 \%$ & 71.25 & $1.24 \pm 13.4 \%$ & 0.030 \\
\hline PGC 23616 & NGC 2590 & 2 & 3.5 & 76.1 & $8.93 \pm 35.7 \%$ & 12.6 & $0.210 \pm 35.6 \%$ & 64.53 & $3.94 \pm 35.6 \%$ & 0.092 \\
\hline PGC 24395 & NGC 2642 & 2 & 4.0 & 21.1 & $8.51 \pm 20.0 \%$ & 13.2 & $0.140 \pm 19.6 \%$ & 55.72 & $2.27 \pm 19.6 \%$ & 0.067 \\
\hline PGC 27351 & NGC 2935 & 2 & 3.0 & 50.1 & $9.07 \pm 23.2 \%$ & 12.4 & $0.185 \pm 18.9 \%$ & 27.63 & $1.49 \pm 18.9 \%$ & 0.050 \\
\hline PGC 28027 & NGC 3001 & 3 & 3.9 & 47.5 & $3 \pm 2.6 \%$ & 16.0 & $0.169 \pm 1.9 \%$ & 30.20 & $1.48 \pm 1.9 \%$ & 0.052 \\
\hline PGC 30108 & NGC 3200 & 2 & 4.5 & 73.0 & $\pm 26.5 \%$ & 12.2 & $0.307 \pm 25.5 \%$ & 44.33 & $3.96 \pm 25.5 \%$ & 0.070 \\
\hline PGC 32026 & NGC 3360 & 4 & 5.0 & 43.6 & $9.88 \pm 19.2 \%$ & 22.1 & $0.076 \pm 19.2 \%$ & 110.28 & $2.44 \pm 19.2 \%$ & 0.063 \\
\hline PGC 33919 & & 2 & 2.6 & 50.2 & $12.02 \pm 21.6 \%$ & 9.4 & $0.128 \pm 20.7 \%$ & 33.60 & $1.25 \pm 20.7 \%$ & 0.046 \\
\hline PGC 34006 & A1108-09 & 2 & 4.8 & 35.6 & $7.21 \pm 23.0 \%$ & 15.5 & $0.142 \pm 22.2 \%$ & 101.65 & $4.20 \pm 22.2 \%$ & 0.070 \\
\hline PGC 42181 & NGC & 3 & 3.8 & 37.6 & $17.51 \pm 13.3 \%$ & 9.7 & $0.073 \pm 11.9 \%$ & 67.61 & $1.44 \pm 11.9 \%$ & 0.033 \\
\hline PGC 45170 & NGC 4939 & 2 & 4.0 & 59.1 & $\pm 11.9 \%$ & 8.6 & $0.144 \pm 13.5 \%$ & 40.11 & $1.68 \pm 13.5 \%$ & 0.026 \\
\hline PGC 45999 & & 2 & 2.2 & 74.9 & $8.07 \pm 24.0 \%$ & 13.9 & $0.263 \pm 22.5 \%$ & 44.59 & $3.41 \pm 22.5 \%$ & 0.076 \\
\hline PGC 46974 & NGC 5135 & 2 & 2.0 & 40.9 & $9.56 \pm 17.3 \%$ & 11.8 & $0.223 \pm 17.3 \%$ & 52.79 & $3.42 \pm 17.3 \%$ & 0.087 \\
\hline PGC 51456 & NGC 5597 & 2 & 6.0 & 27.4 & $14.83 \pm 6.6 \%$ & 7.7 & $0.090 \pm 6.6 \%$ & 33.99 & $0.89 \pm 6.6 \%$ & 0.039 \\
\hline PGC 53499 & NGC 5792 & 2 & 3.0 & 80.5 & $16.26 \pm 38.7 \%$ & 7.0 & $0.381 \pm 36.6 \%$ & 25.67 & $2.84 \pm 36.6 \%$ & 0.054 \\
\hline PGC 54250 & NGC 5833 & 2 & 4.2 & 67.2 & $\pm 37.4 \%$ & 19.2 & $0.379 \pm 36.1 \%$ & 38.28 & $4.22 \pm 36.1 \%$ & 0.109 \\
\hline PGC 55738 & NGC 5968 & 2 & 2.0 & 36.1 & $6.24 \pm 68.5 \%$ & 17.8 & $0.225 \pm 66.6 \%$ & 67.44 & $4.41 \pm 66.6 \%$ & 0.096 \\
\hline PGC 56630 & IC 4585 & 2 & 3.0 & 72.4 & $32.19 \pm 29.1 \%$ & 3.6 & $0.074 \pm 28.4 \%$ & 46.64 & $1.00 \pm 28.4 \%$ & 0.028 \\
\hline PGC 57924 & NGC 6118 & 2 & 6.0 & 69.7 & $12.68 \pm 24.4 \%$ & 9.0 & $0.269 \pm 23.4 \%$ & 21.63 & $1.69 \pm 23.4 \%$ & 0.052 \\
\hline PGC 59325 & IC 46 & 2 & 4.1 & 50.7 & $9.58 \pm 19.7 \%$ & 11.8 & $0.128 \pm 14.6 \%$ & 38.23 & $1.42 \pm 14.6 \%$ & 0.067 \\
\hline PGC 60907 & IC 4664 & 2 & 3.2 & 50.9 & $13.85 \pm 43.3 \%$ & 8.2 & $0.096 \pm 41.9 \%$ & 65.25 & $1.82 \pm 41.9 \%$ & 0.048 \\
\hline PGC 61315 & NGC 6492 & 2 & 3.8 & 55.0 & $10.62 \pm 38.7 \%$ & 10.7 & $0.164 \pm 38.6 \%$ & 55.60 & $2.65 \pm 38.6 \%$ & 0.062 \\
\hline PGC 63204 & IC 4852 & 2 & 4.5 & 31.7 & $6.89 \pm 22.6 \%$ & 16.2 & $0.151 \pm 19.1 \%$ & 59.64 & $2.62 \pm 19.1 \%$ & 0.093 \\
\hline PGC 64042 & IC 4933 & 2 & 4.2 & 31.7 & $15.12 \pm 10.3 \%$ & 7.5 & $0.121 \pm 9.1 \%$ & 64.04 & $2.25 \pm 9.1 \%$ & 0.053 \\
\hline PGC 64168 & NGC 6862 & 2 & 3.5 & 46.2 & $10.82 \pm 17.6 \%$ & 10.5 & $0.136 \pm 15.5 \%$ & 55.28 & $2.19 \pm 15.5 \%$ & 0.082 \\
\hline PGC 64413 & NGC 6872 & 2 & 3.0 & 70.0 & $8.50 \pm 35.3 \%$ & 13.2 & $0.484 \pm 29.6 \%$ & 62.75 & $8.83 \pm 29.6 \%$ & 0.077 \\
\hline PGC 65125 & NGC 6937 & 2 & 4.9 & 49.9 & $9.06 \pm 33.1 \%$ & 12.5 & $0.185 \pm 28.7 \%$ & 61.64 & $3.32 \pm 28.7 \%$ & 0.079 \\
\hline PGC 67417 & NGC 7125 & 2 & 5.0 & 31.3 & $6.04 \pm 20.8 \%$ & 18.3 & $0.077 \pm 18.8 \%$ & 39.59 & $0.89 \pm 18.8 \%$ & 0.025 \\
\hline
\end{tabular}


Table 1. continued

\begin{tabular}{|c|c|c|c|c|c|c|c|c|c|c|}
\hline$\overline{\mathrm{PGC}}$ & Names & $m$ & $\bar{T}$ & $\begin{array}{c}\gamma \\
\left({ }^{\circ}\right)\end{array}$ & $\Lambda \pm \mathrm{d} \Lambda / \Lambda$ & $\begin{array}{c}\mu \\
\left(^{\circ}\right)\end{array}$ & $h \pm \underset{\left({ }^{\prime}\right)}{h} h / h$ & $\begin{array}{c}d \\
(\mathrm{Mpc})\end{array}$ & $\begin{array}{c}H \pm \mathrm{d} H / H \\
(\mathrm{kpc})\end{array}$ & $H / D_{0}$ \\
\hline$\overline{\text { PGC } 67418}$ & NGC 7126 & 2 & 5.0 & 62.8 & $8.15 \pm 23.4 \%$ & 13.8 & $0.224 \pm 22.8 \%$ & 39.68 & $2.58 \pm 22.8 \%$ & 0.078 \\
\hline PGC 67532 & NGC 7141 & 2 & 4.0 & 43.6 & $13.50 \pm 13.2 \%$ & 8.4 & $0.210 \pm 12.8 \%$ & 38.59 & $2.36 \pm 12.8 \%$ & 0.050 \\
\hline PGC 69183 & NGC 7309 & 3 & 5.0 & 21.1 & $16.64 \pm 11.1 \%$ & 10.2 & $0.083 \pm 10.7 \%$ & 54.83 & $1.32 \pm 10.7 \%$ & 0.041 \\
\hline PGC 69453 & NGC 7329 & 2 & 3.0 & 51.7 & $7.99 \pm 15.6 \%$ & 14.1 & $0.298 \pm 15.6 \%$ & 40.76 & $3.53 \pm 15.6 \%$ & 0.077 \\
\hline PGC 69507 & & 2 & 5.0 & 30.3 & $16.59 \pm 12.2 \%$ & 6.9 & $0.061 \pm 11.9 \%$ & 144.28 & $2.56 \pm 11.9 \%$ & 0.040 \\
\hline PGC 70027 & NGC 7412 & 2 & 3.0 & 47.9 & $16.37 \pm 14.2 \%$ & 7.0 & $0.191 \pm 13.6 \%$ & 22.67 & $1.26 \pm 13.6 \%$ & 0.049 \\
\hline PGC 70096 & NGC 7424 & 2 & 6.0 & 31.7 & $6.59 \pm 7.1 \%$ & 16.9 & $0.215 \pm 9.6 \%$ & 12.39 & $0.77 \pm 9.6 \%$ & 0.023 \\
\hline PGC 70800 & NGC 7531 & 2 & 4.0 & 63.5 & $9.30 \pm 13.4 \%$ & 12.1 & $0.317 \pm 11.2 \%$ & 20.92 & $1.93 \pm 11.2 \%$ & 0.069 \\
\hline PGC 70884 & NGC 7552 & 2 & 2.0 & 37.4 & $13.83 \pm 4.9 \%$ & 8.2 & $0.204 \pm 4.9 \%$ & 20.91 & $1.24 \pm 4.9 \%$ & 0.059 \\
\hline PGC 71001 & NGC 7582 & 2 & 2.0 & 63.0 & $12.86 \pm 16.6 \%$ & 8.8 & $0.278 \pm 16.7 \%$ & 20.68 & $1.67 \pm 16.7 \%$ & 0.055 \\
\hline PGC 72444 & NGC 7755 & 2 & 4.7 & 45.7 & $6.45 \pm 16.0 \%$ & 17.2 & $0.288 \pm 15.9 \%$ & 39.63 & $3.32 \pm 15.9 \%$ & 0.076 \\
\hline PGC 72718 & & 2 & 4.7 & 39.5 & $8.99 \pm 13.1 \%$ & 12.5 & $0.113 \pm 13.1 \%$ & 102.64 & $3.37 \pm 13.1 \%$ & 0.088 \\
\hline PGC 72929 & & 2 & 3.7 & 46.2 & $8.40 \pm 18.0 \%$ & 13.4 & $0.135 \pm 16.0 \%$ & 63.11 & $2.48 \pm 16.0 \%$ & 0.102 \\
\hline
\end{tabular}

Table 2. The regression coefficients

\begin{tabular}{lccccc}
\hline & $N$ & $a$ or $a^{\prime}$ & $b$ or $b^{\prime}$ & $r$ & $\begin{array}{c}r_{\alpha} \\
(\alpha=1 \%)\end{array}$ \\
\hline Fig. 1 & 70 & $1.19 \pm 0.14$ & $-1.93 \pm 0.08$ & 0.708 & 0.302 \\
Fig. 2 & 70 & $1.20 \pm 0.25$ & $-0.35 \pm 0.14$ & 0.504 & 0.302 \\
Fig. 3 & 77 & $1.24 \pm 0.16$ & $-1.98 \pm 0.09$ & 0.665 & 0.288 \\
Fig. 4 & 77 & $1.35 \pm 0.24$ & $-0.47 \pm 0.14$ & 0.539 & 0.288 \\
\hline
\end{tabular}

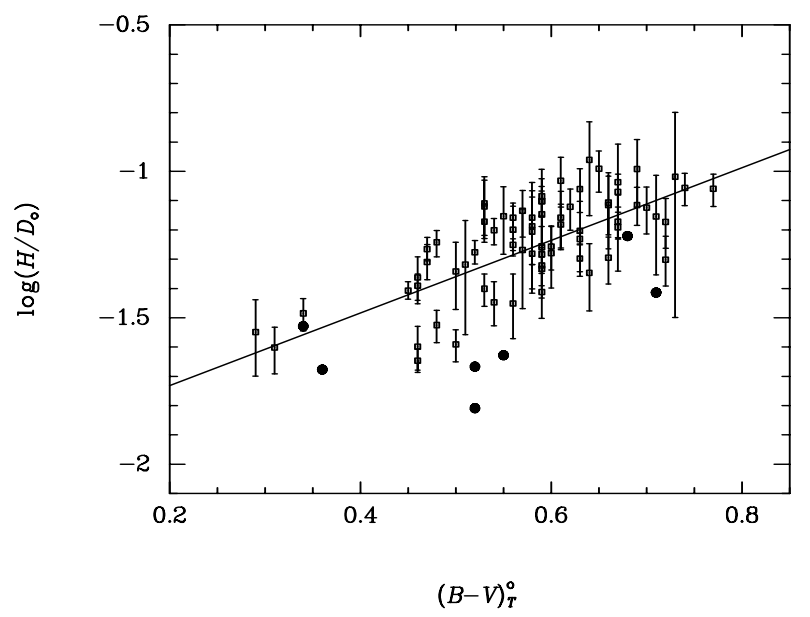

Fig. 3. Flatness of spiral galaxy plotted versus the corrected $B-V$ color, the data (black circle) are from van der Kruit and Searle's papers

The regression coefficients, and their errors are given in Table 2. $r$ in Col. 5 of Table 2 is the correlation coefficient and $r_{\alpha}(\alpha=0.01)$ the lowest correlation coefficient.

From Figs. 1 and 3, one could see a trend that flatter galaxies are bluer, and the strong correlation is encouraging. A similar tendency can be found from Figs. 2 and 4 that the smaller the scaleheight of a galaxy is, the bluer

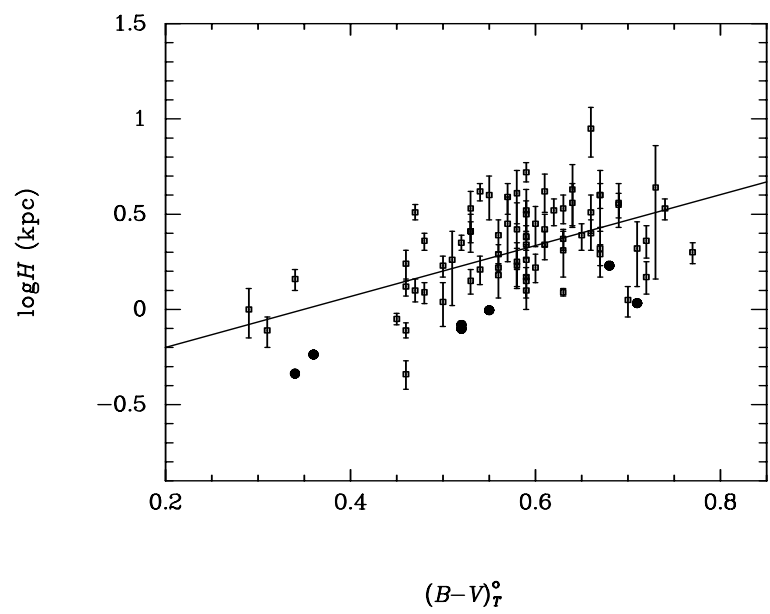

Fig. 4. Scaleheight of spiral galaxy plotted versus the corrected $B-V$ color, the data (black circle) are from van der Kruit and Searle's papers

the galaxy is. Figure 5 plots flatness of spiral galaxy as a function of the corrected $U-B$ color $\left((U-B)_{T}^{0}\right)$, which the total color index taken from RC3 corrected for differential galactic and internal extinction (to "face-on") and for redshift between $U$ and $B$ bands. The dependence of scaleheight of spiral on $(U-B)_{T}^{0}$ is illustrated in Fig. 6 . 


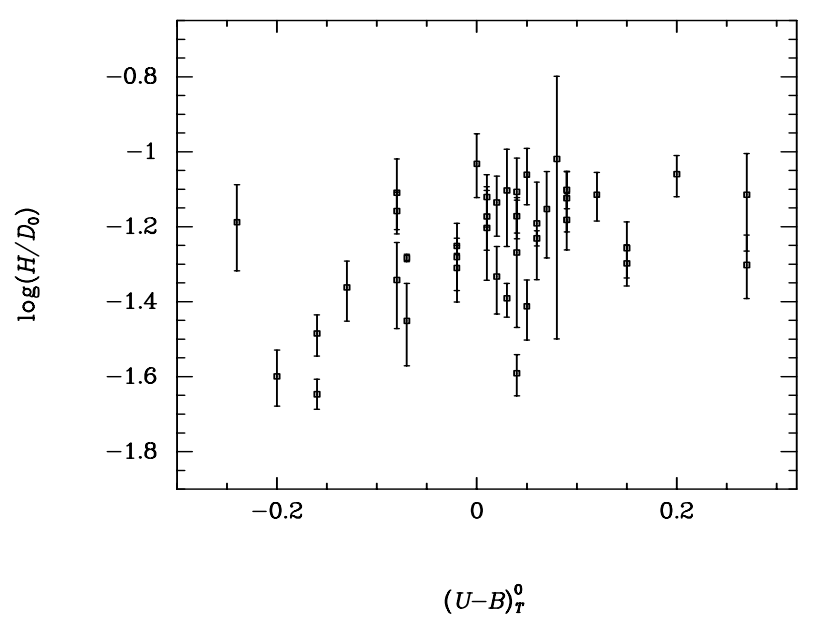

Fig. 5. Flatness of spiral galaxy plotted versus the corrected $U-B$ color

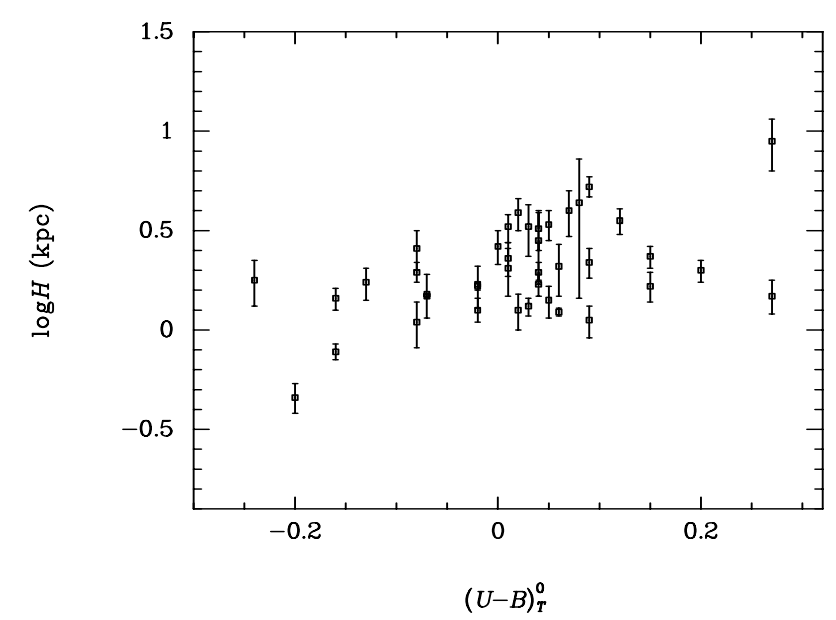

Fig. 6. Scaleheight of spiral galaxy plotted versus the corrected $U-B$ color

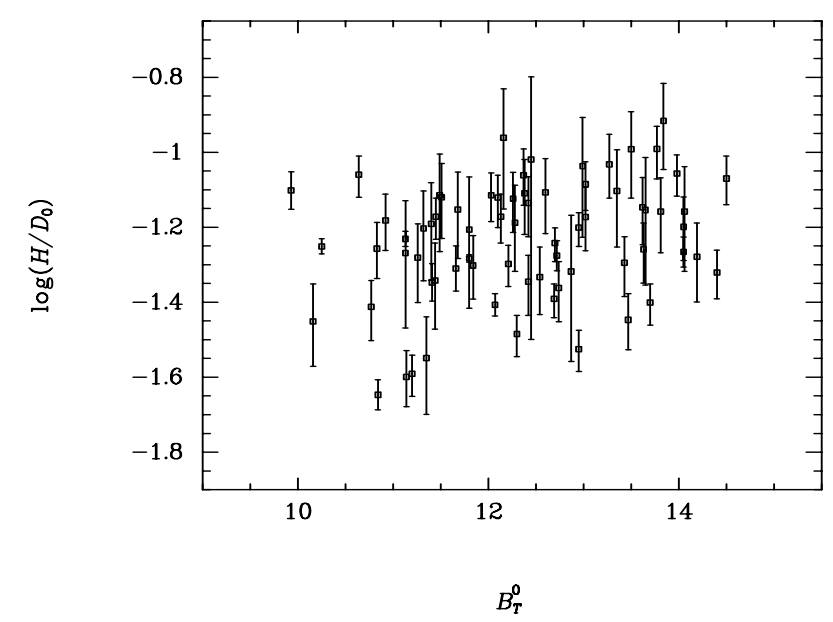

Fig. 7. Flatness of spiral galaxy plotted versus the total magnitude in the $B$ system

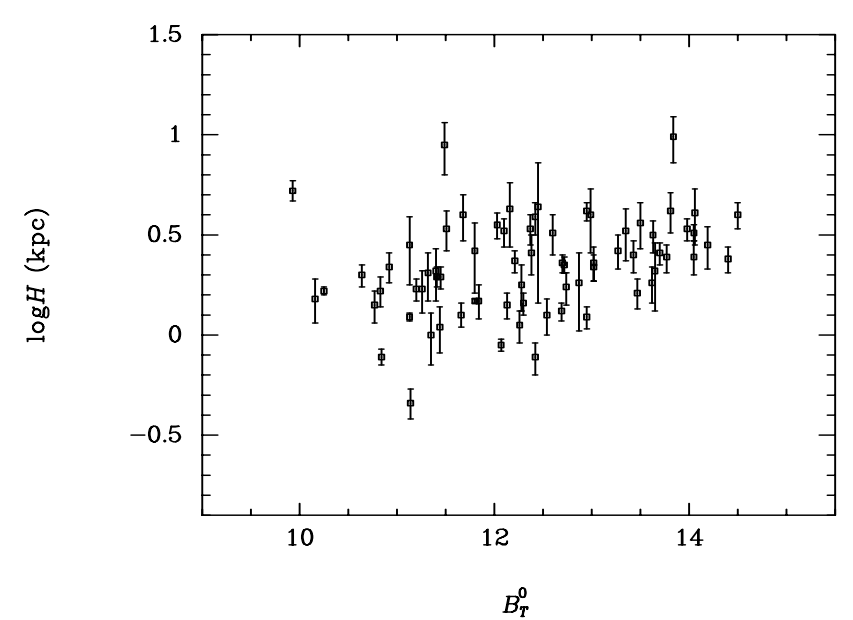

Fig. 8. Scaleheight of spiral galaxy plotted versus the total magnitude in the $B$ system

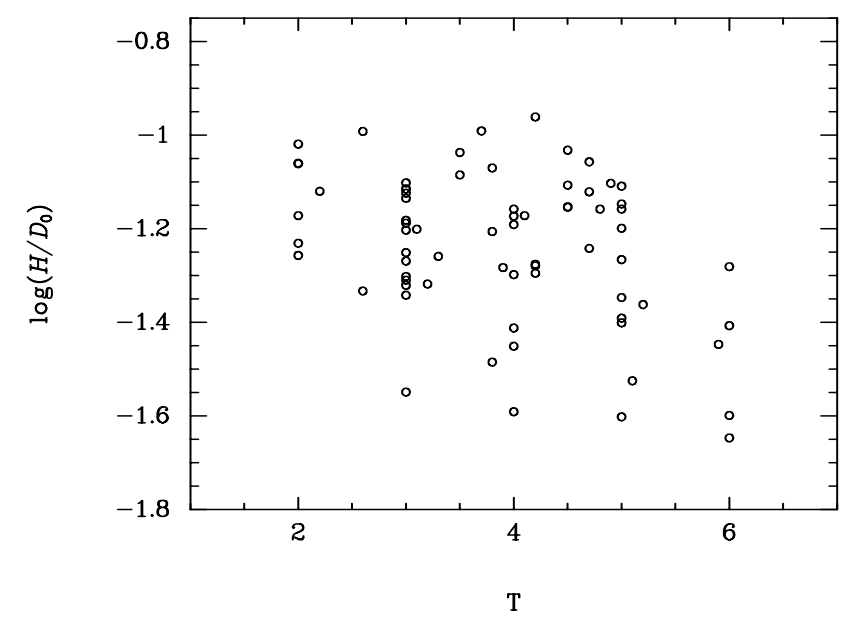

Fig. 9. Flatness of spiral galaxy plotted versus the Hubble sequence

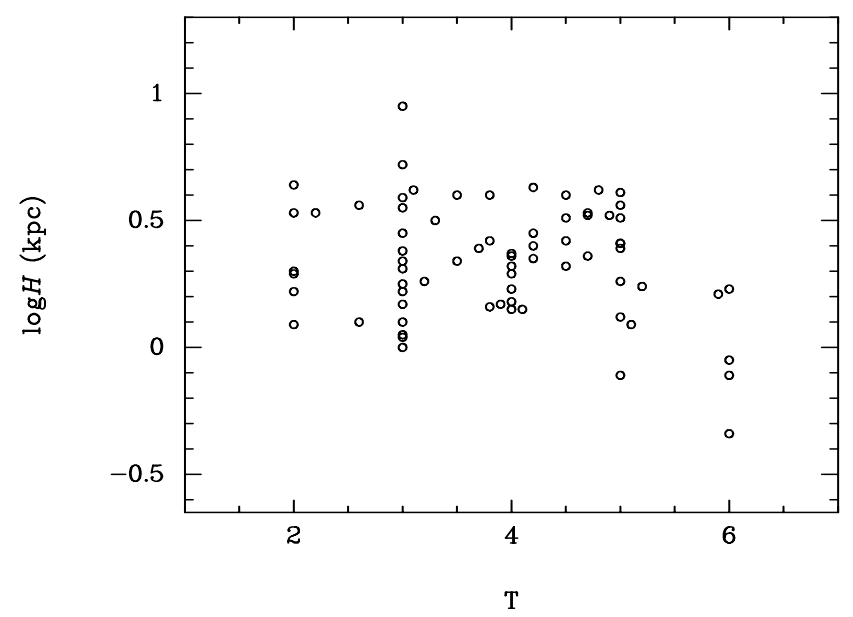

Fig. 10. Scaleheight of spiral galaxy plotted versus the Hubble sequence 


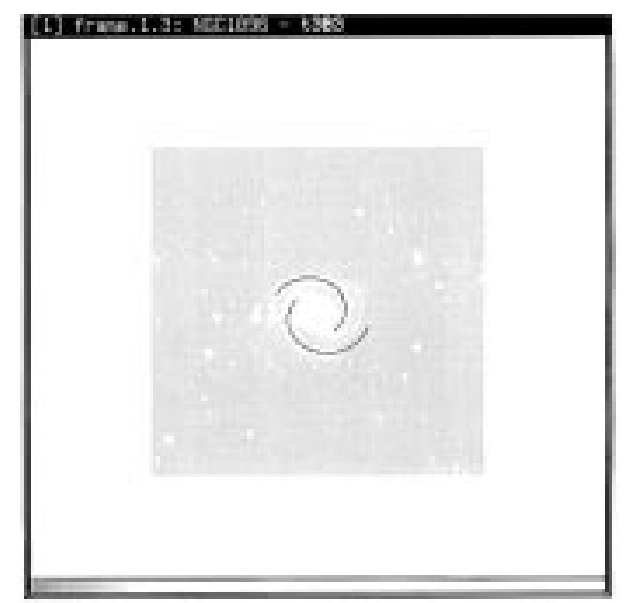

Fig. 11. The image of NGC 1096 and with superimposed fitting logarithmic spiral curves

\section{Dependence on magnitude.}

Figure 7 shows the correlation between flatness of spiral galaxy and the total "face-on" magnitude $B_{T}^{0}$, taken from RC3, corrected for Galactic and internal absorption and for redshift in the $B$ system. The correlation of scaleheight of spiral with $B_{T}^{0}$ is shown in Fig. 8. It is interesting to find that the flatter galaxies look brighter, and that the smaller the scaleheight of a galaxy is, the brighter the galaxy looks.

\section{Dependence on Hubble type.}

The tightness of the spiral pattern, in addition to the disk resolution and bulge-to-disk ratio, are the fundamental criteria in Hubble's (1926) classification of spirals. It would be suggestive to see the dependence of flatness of spiral galaxies on the Hubble types, which is shown in Fig. 9. Although the scatter is quite significant, one can still find a trend that spirals become flatter along the Hubble types Sab - Scd. Part of this scatter can be attributed to the estimated dispersion of flatness among the individual galaxies themselves. An additional dispersion of comparable magnitude is expected from the discrete binning of the measured Hubble types. Kennicutt (1981) has already noted that different Hubble classifications based on different weighting of arm morphology of disk resolution will lead to inconsistencies if the data sets are indiscriminately mixed. On the other hand, we have not found any correlation of scaleheights of spirals with $T$, as Fig. 10 indicates.

\section{Summary and conclusion}

The primary purpose of this paper is to estimate scaleheights of 486 southern spiral galaxies by Peng's method and to explore the correlation between the flatness of a spiral galaxy and the color index. At the same time, we found some other statistical correlation between flatness of spiral galaxy and the total "face-on" magnitude in the $B$ system, and so on. Our main conclusions are that flatter galaxies are bluer and look brighter and that spirals become flatter along the Hubble sequence Sab - Scd.

Acknowledgements. We would like to thank Prof. Jing-yao Hu for his hospitality and discussion at Xinglong Station of Beijing Astronomical Observatory, and are grateful to Prof. Jie-hao Huang for his much help in English and operating computer. We acknowledge Dr. Qiu-sheng Gu for his much help in operating computer. This work is supported by both the National Nature Science Foundation, National Grand Project "Climbing Up" of China and the Doctoral programm Foundation of State Education Commission.

\section{Appendix}

In Table A1, we list the scaleheights of 416 spiral galaxies measured based on Peng's method (1988), the value of the inclination is $\arccos \left(d_{25} / D_{25}\right)$. We do not compare the fitted spiral arms with the images. Type the mean revised morphological type, $S_{\mathrm{R}}$ the standard deviation discussed in Peng's paper (1988).

\section{References}

de Vaucouleurs G., de Vaucouleurs A., Corwin H.G., Buta B.J., Fouque P, 1991, the Third Reference Catalog of Bright Galaxies. New York: Springer-Verlag

Elmegreen D.M., Elmegreen B.G., 1987, ApJ 314, 3

Hubble E.P., 1926, ApJ 64, 321

Kennicutt R.C., 1981, ApJ 86, 1847

Peng Qiu-he, 1988, A\&A 206, 18

van der Kruit P.C., Searle L., 1981, A\&A 95, 105

van der Kruit P.C., Searle L., 1982, A\&A 110, 79 